\title{
PRIMARY MALIGNANT MIXED MULLERIAN TUMOUR OF THE CERVIX: A RARE ENTITY
}

\author{
Ashwini Karthick1, Kanchana Madurai Padmanaban²
}

1 Post Graduate, Department of Pathology, Institute of Obstetrics and Gynaecology, Madras Medical College, Chennai. 2 Professor, Department of Pathology, Institute of Obstetrics and Gynaecology, Madras Medical College, Chennai.

\begin{abstract}
Malignant mixed Mullerian tumour of the cervix is a rare entity. One such case of primary malignant mixed Mullerian tumour of the cervix is being presented in this article. It occurred in a 65-year-old female and presented as a polypoidal mass. Microscopically, epithelial and mesenchymal components were made out. Immunohistochemical analysis was done with cytokeratin and vimentin. The patient was treated with radical radiotherapy and is free from recurrence at the end of three years of treatment.
\end{abstract}

\section{KEYWORDS}

Metaplasia, Bidirectional Differentiation, HPV-16.

HOW TO CITE THIS ARTICLE: Karthick A, Padmanaban KM. Primary malignant mixed Mullerian tumour of the cervix: a rare entity. J. Evolution Med. Dent. Sci. 2016;5(56):3882-3884, DOI: 10.14260/jemds/2016/889

\section{INTRODUCTION}

Malignant mixed Mullerian tumour of the cervix is extremely rare. It accounts for $0.005 \%$ of all the malignancies of the cervix. Malignant mixed Mullerian tumour of the cervix was first described by Ferriera and Colleagues in the year $1951 .^{1}$ About 50 cases have been reported in the literature so far. It typically occurs in post-menopausal women. They are composed of malignant epithelial and sarcomatous components. ${ }^{2}$ Synonyms include carcinosarcoma, malignant mesodermal mixed tumour and metaplastic carcinoma.

\section{CASE REPORT}

A 65-year-old female, P4 L4 came with complaints of watery discharge per vaginum. She attained menopause 20 years back. On speculum examination, cervix was found to be replaced by the growth. Firm, irregular growth occupying the cervix and upper third of vagina was noted in per-vaginal examination. Per-rectal examination showed bilateral parametrial involvement. Cystoscopy was normal.

USG abdomen and pelvis showed a growth in the cervix measuring $6.5 * 5 \mathrm{~cm}$ with a pyometra. CT abdomen and pelvis showed an ill-defined irregularly enhancing mass centered in the cervix obliterating the endocervical canal measuring $5.9 * 2.8 \mathrm{~cm}$ with direct extension of mass up to lower-third of vagina and an ill-defined nodule of $1.6 \mathrm{~cm}$ in the left vulva. Bilateral parametrial involvement was present. Myometrium and endometrium were uninvolved. Large pyometra measuring $4.8 \mathrm{~cm}$ in thickness was noted. Urinary bladder, rectum, pelvic side walls were uninvolved. Necrotic left iliac node measuring $9 \mathrm{~mm}$ was noted.

Biopsy was taken from the growth. Microscopically, the sections showed a neoplasm composed of malignant squamous epithelial cells arranged in syncytial sheets. The stroma was composed of neoplastic spindle shaped cells arranged in fascicles.

Financial or Other, Competing Interest: None.

Submission 06-06-2016, Peer Review 29-06-2016,

Acceptance 07-07-2016, Published 14-07-2016.

Corresponding Author:

Dr. Ashwini Karthick,

Door No. E1,

Euphoria Apartments,

Chittibabu Nagar, 3rd Cross Street,

Pallikaranai,

Chennai-600100.

E-mail: dr.achu15@gmail.com

DOI: $10.14260 /$ jemds/2016/889
Mitotic figures and tumour giant cells were also seen. Immunohistochemistry showed cytokeratin positivity in the carcinomatous and sarcomatous tumour cells and vimentin positivity in the sarcomatous components. Smooth muscle actin positivity was seen only around the blood vessels.

The patient was a diabetic and hypertensive and was a known case of coronary artery disease on medication. Taking these into account, she was treated with palliative radiotherapy - 28 cycles with 50 cGy and oral chemotherapy with doxifluridine $200 \mathrm{mg}$ BD.

Follow-up of the patient after completion of radiotherapy showed no evidence of growth in the cervix. The patient was followed-up for three years and found to be free from recurrence.

\section{DISCUSSION}

The most common site for malignant mixed Mullerian tumour in the female genital tract is the uterine corpus. Primary malignant mixed Mullerian tumour of the cervix is extremely rare. The most common age group for cervical malignant mixed Mullerian tumour is 65 years, though the age range varies from 12 to 93 years.

It typically occurs in post-menopausal women. Clinical features include vaginal bleeding, abnormal vaginal cytology and polypoid cervical mass, though in our case the presenting complaint was foul smelling watery discharge per vaginum. Malignant mixed Mullerian tumours of the cervix are aggressive, known to recur and have a poor prognosis.

Grossly, the cervical tumours ranged between 1.1 and 10 $\mathrm{cm}$ in size. Cervical malignant mixed Mullerian tumours present as polypoidal or pedunculated masses. Microscopically, they are composed of admixture of malignant epithelial and mesenchymal components exhibiting a biphasic appearance. ${ }^{3}$ The epithelial component represents a variety of different histological subtypes, either alone or in combination, which includes squamous cell carcinoma, basaloid squamous carcinoma, adenocarcinoma, adenosquamous carcinoma, adenoid basal carcinoma, adenoid cystic carcinoma and undifferentiated carcinoma. Most common pattern is the basaloid pattern consisting of anastomosing densely cellular trabeculae composed of small cells with scant cytoplasm and peripheral palisading.

The sarcomatous component may be homologous (Fibrosarcoma/endometrial stromal sarcoma) or heterologous (Chondrosarcoma/rhabdomyosarcoma). 
The sarcomatous components are usually of high grade with or without myxoid changes. One case report described neuroendocrine component in malignant mixed Mullerian tumour of the cervix.

Recent clinicopathologic, immunohistochemical and molecular genetic studies have proved that most malignant mixed Mullerian tumours likely represent carcinomas with mesenchymal components as a consequence of metaplasia and tumour progression. They can also arise by a process of bidirectional differentiation of a multipotent stem cell.

On immunohistochemical analysis, both epithelial and sarcomatous components of malignant mixed Mullerian tumour show positivity for broad-spectrum cytokeratins, High Molecular Weight Cytokeratins (HMWCK), Low Molecular Weight Cytokeratins (LMWCK) and Epithelial Membrane Antigen (EMA). The sarcomatous components show positivity for vimentin, desmin, Muscle Specific Actin (MSA) and Smooth Muscle Actin (SMA). ${ }^{4}$

An occasional case was associated with prior radiation. In a study of eight patients with cervical malignant mixed Mullerian tumour, HPV-DNA was detected by Polymerase Chain Reaction (PCR) in all cases. Grayson et al performed insitu hybridization studies, which showed the presence of HPVDNA in both epithelial and sarcomatous components in three cases. 5 These observations indicate the metaplastic theory of histogenesis. HPV-16 is most commonly involved followed by HPV-18.

The differential diagnosis for cervical malignant mixed Mullerian tumour includes extension from the tumour in the uterine corpus. Due to the rarity of this tumour, no evidence based management guidelines are available. Surgery is the principal modality of treatment. Malignant mixed Mullerian tumours are usually treated by radical hysterectomy followed by chemotherapy with or without radiotherapy. Radical radiotherapy with or without chemotherapy is recommended for locally advanced disease. Patients with metastatic disease are treated with palliative chemotherapy. The clinical behaviour of these tumours are dominated by the carcinomatous component. Prognosis is better for small polypoidal masses. ${ }^{6}$

\section{SUMMARY}

Cervical malignant mixed Mullerian tumours are rare and they represent carcinomas with mesenchymal component as a consequence of metaplasia. They present as polypoidal masses in post-menopausal women and are associated with Human Papillomavirus infection. They behave aggressively and extension from uterine malignant mixed Mullerian tumour should be excluded.

Correct diagnosis is based on dominant location of the tumour, appearance of the carcinomatous component and detection of Human Papillomavirus.

Fourteen cases of malignant mixed Mullerian tumour have been reported in our institution in the past six years of which seven occurred in the endometrium, four in the ovaries, one in the fallopian tube and two in the cervix (One lost for followup). Hence, this case is being presented here for its rarity.

\section{PRIMARY MALIGNANT MIXED MULLERIAN TUMOUR OF THE CERVIX - IMAGES}

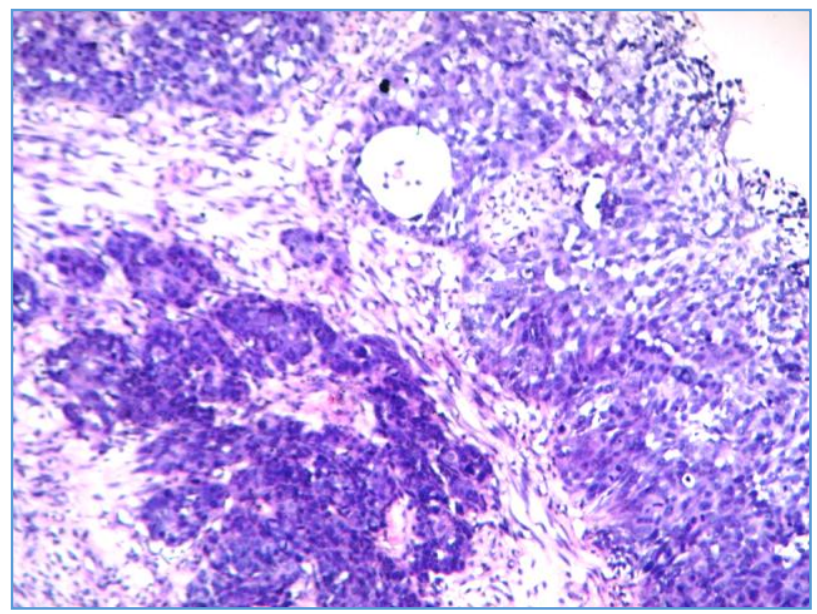

Fig. 1: Shows Sheets of Malignant Squamous Epithelial Cells; 400x, H\&E

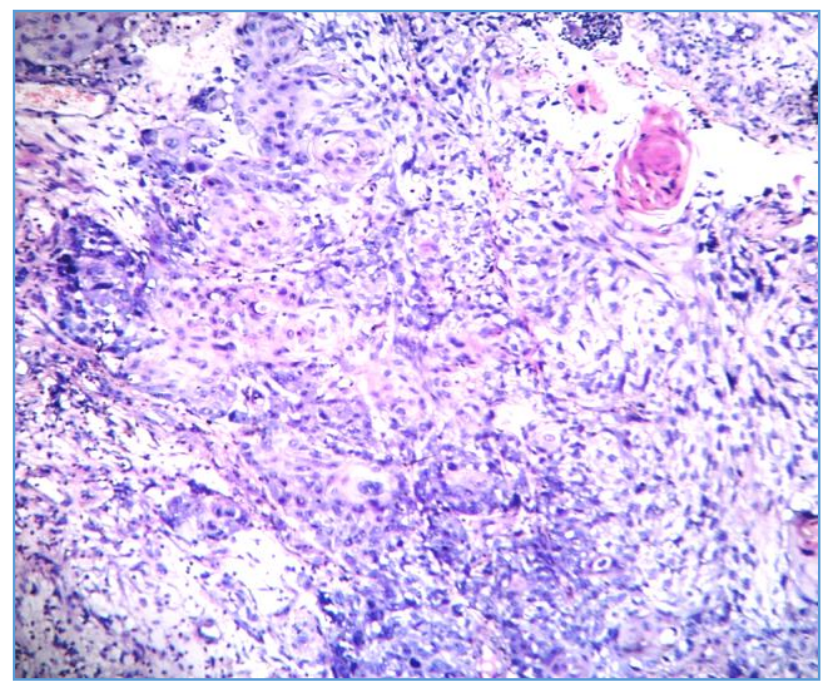

Fig. 2: Shows Malignant Squamous Epithelial Cells Exhibiting Keratinisation; 400X, $\mathrm{H} \& \mathrm{E}$

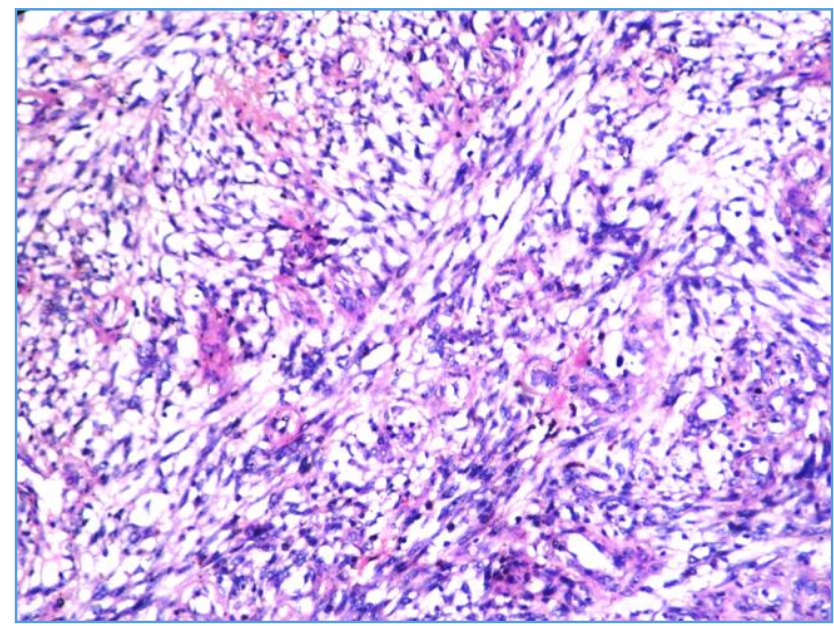

Fig. 3: Shows Sarcomatous Component Comprising Fascicles of Spindle Shaped Cells; 400x, H\&E 


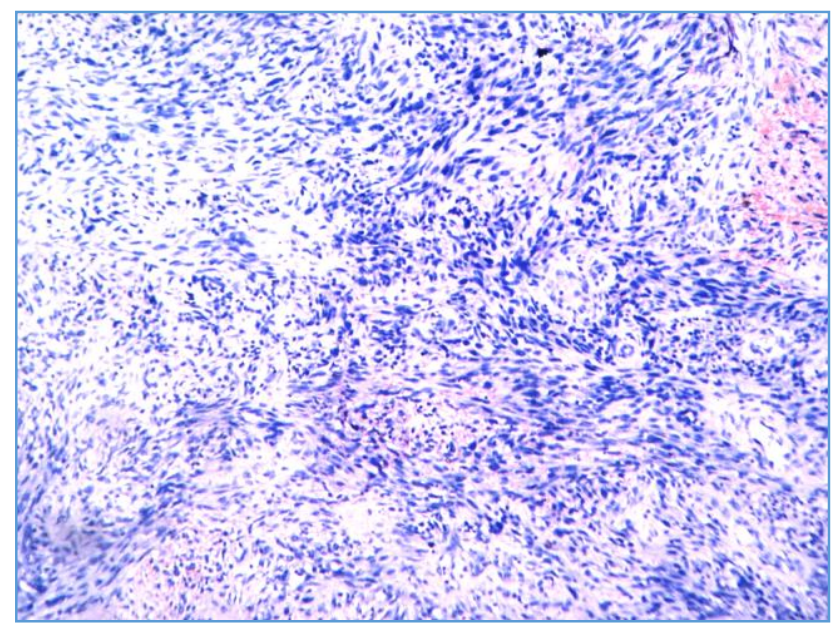

Fig. 4: Shows Fascicles of Spindle Shaped Cells; 400x, H\&E

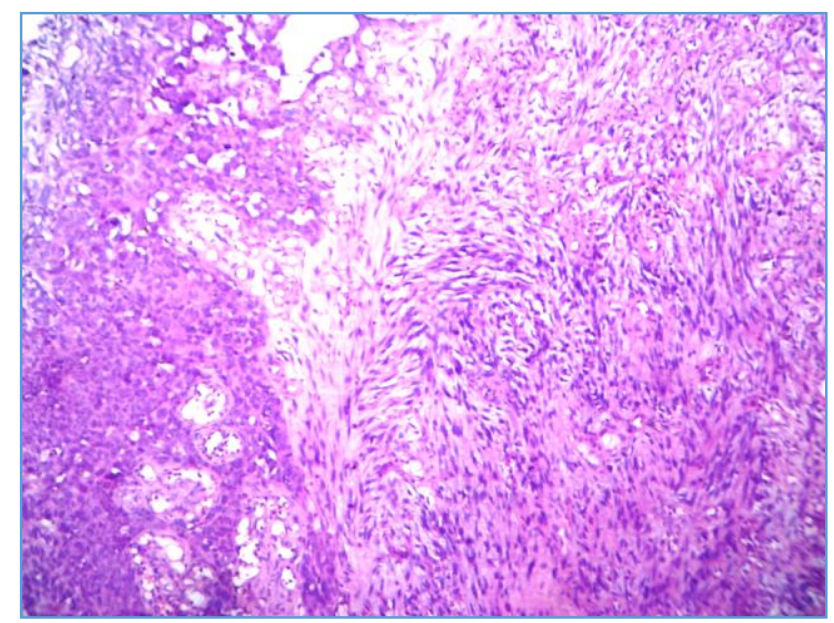

Fig. 5: Shows Biphasic Population of Tumour Cells Composed of Malignant Squamous Epithelial Component and Spindle Shaped Sarcomatous Component; 400x, $\mathrm{H \& E}$

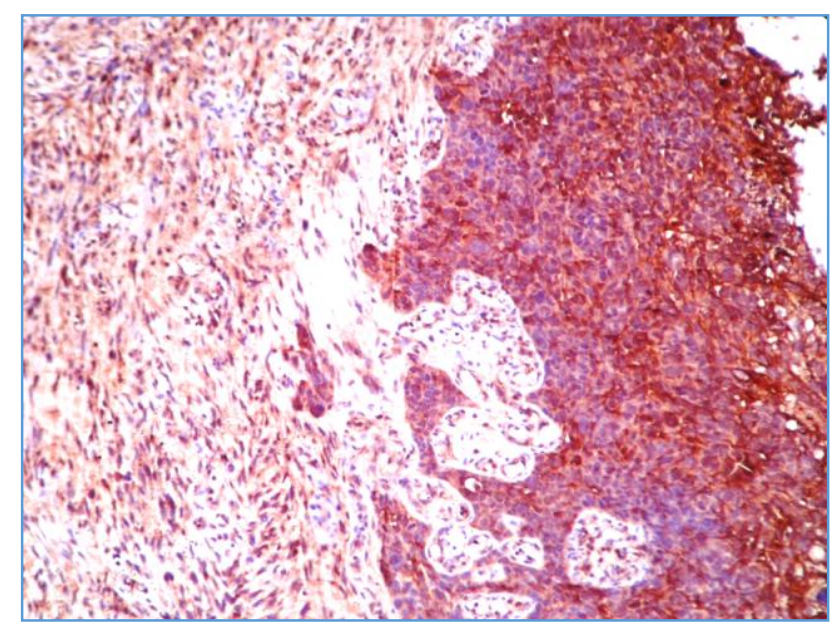

Fig. 6: Immunohistochemistry shows Cytokeratin Positivity in Malignant Squamous and Spindle Cells; 400x

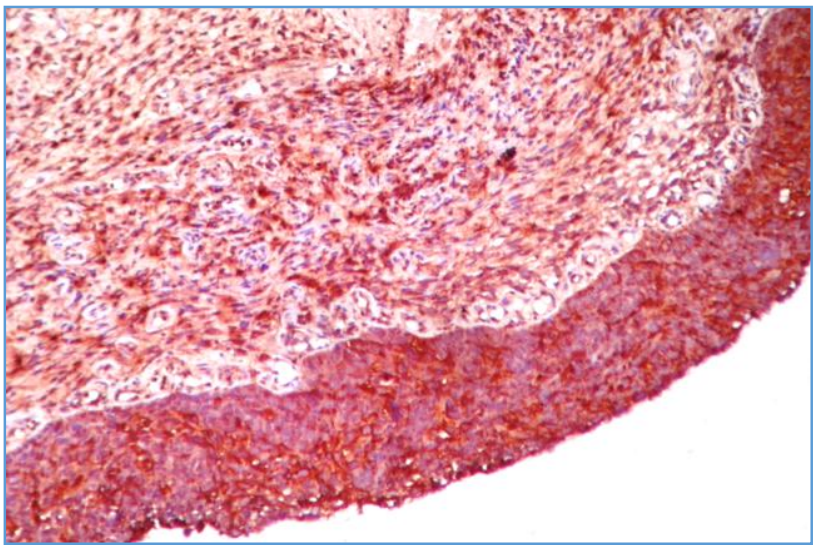

Fig. 7: Immunohistochemistry shows Cytokeratin Positivity in Malignant Squamous and Spindle Cells, 400x

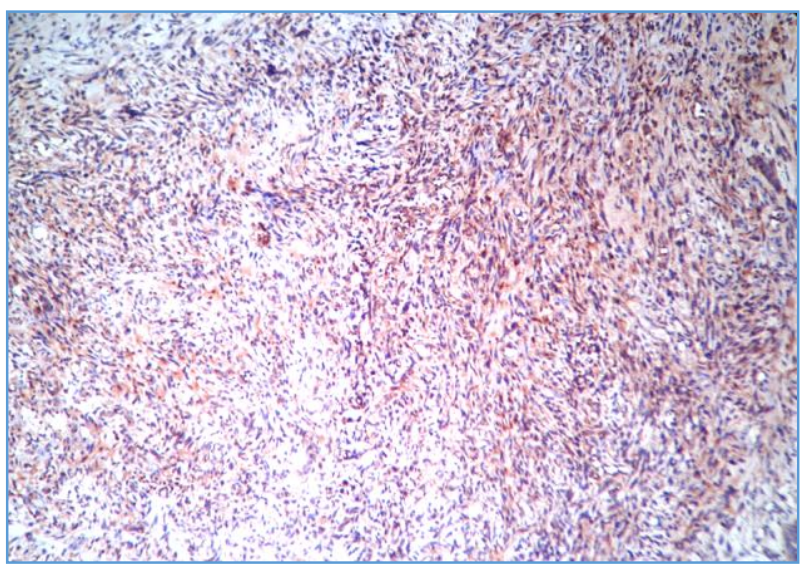

Fig. 8: Immunohistochemistry shows Vimentin Positivity in Spindle Shaped Cells, 100x

\section{CONCLUSION}

Cervical malignant mixed Mullerian tumours are rare malignancies that may at times pose diagnostic difficulties to the clinician. Accurate diagnosis is essential for appropriate treatment and prognostication of the disease.

\section{REFERENCES}

1. Ferriera HP. A case of mixed mesodermal tumour of the cervix. J Obstet Gynaecol Br Emp 1951;58:446-448.

2. Abidi A, Menn K, Sherman A, et al. Cervical carcinosarcoma: a case report. J Reprod Med 2008;53(2):138-40.

3. Clement PB, Zubovitus JT, Young RH, et al. MMMT of uterine cervix, a report of 9 cases of a neoplasm with morphology often different from its counterpart in the corpus. Int J Gynaecol Pathol 1998;17(3): 211-22.

4. Maheshwari A, Gupta S, Shet A, et al. Diagnostic dilemma in a case of MMMT of the cervix. World J Surg Oncol 2006;4:36.

5. Grayson W, Taylor LF, Cooper K. Carcinosarcomas of the uterine cervix: a report of eight cases with immunohistochemical analysis and evaluation of HPV status. Am J Surg Pathol 2001;25(3):338-47.

6. Kuyumcuoglu U, Kale A. Homologous type of malignant mixed mullerian tumour of the uterus presenting as a cervical mass. J Chin Med Assoc 2009;72(10):533-35. 